\title{
GROND coverage of the main peak of gamma-ray burst $130925 A^{\star}, \star \star$
}

\author{
J. Greiner ${ }^{1,2}$, H.-F. Yu ${ }^{1,2}$, T. Krühler ${ }^{3}$, D. D. Frederiks ${ }^{4}$, A. Beloborodov ${ }^{5}$, P. N. Bhat ${ }^{6}$, J. Bolmer $^{7,1}$ \\ H. van Eerten ${ }^{1, \star \star \star}$, R. L. Aptekar ${ }^{4}$, J. Elliott ${ }^{1}$, S. V. Golenetskii ${ }^{4}$, J. F. Graham ${ }^{1}$, K. Hurley ${ }^{8}$, D. A. Kann ${ }^{1,9}$, S. Klose $^{9}$, \\ A. Nicuesa Guelbenzu ${ }^{9}$, A. Rau ${ }^{1}$, P. Schady ${ }^{1}$, S. Schmidl ${ }^{9}$, V. Sudilovsky ${ }^{1}$, D. S. Svinkin ${ }^{4}$, M. Tanga ${ }^{1}$, M. V. Ulanov ${ }^{4}$, \\ K. Varela ${ }^{1}$, A. von Kienlin ${ }^{1}$, and X.-L. Zhang ${ }^{1}$
}

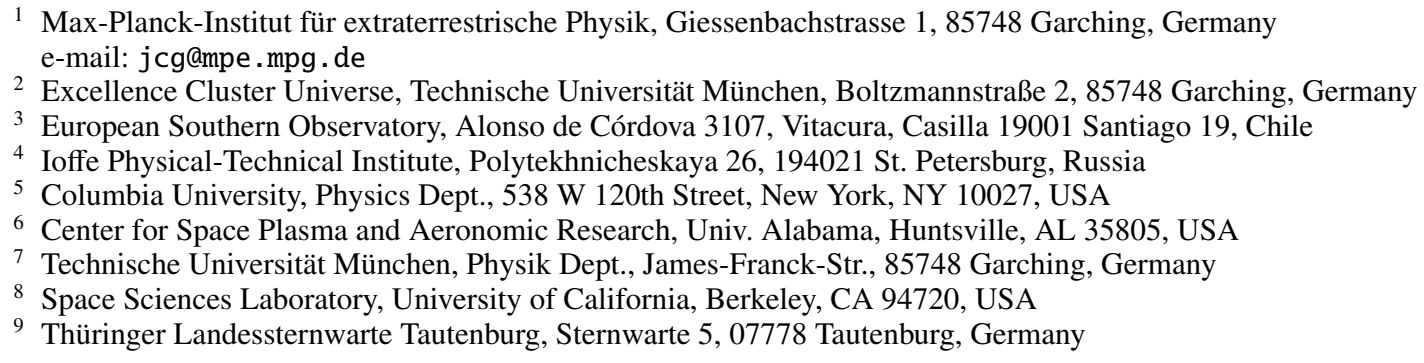

Received 22 May 2014 / Accepted 11 July 2014

\section{ABSTRACT}

\begin{abstract}
Aims. Prompt or early optical emission in gamma-ray bursts (GRBs) is notoriously difficult to measure, and observations of the dozen cases show a large variety of properties. Yet, such early emission promises to help us achieve a better understanding of the GRB emission process(es).

Methods. We performed dedicated observations of the ultra-long duration (T90 about $7000 \mathrm{~s}$ ) Swift GRB 130925A in the optical/nearinfrared with the 7-channel Gamma-Ray burst Optical and Near-infrared Detector (GROND) at the 2.2 m MPG/ESO telescope.

Results. We detect an optical/near-infrared flare with an amplitude of nearly 2 mag which is delayed with respect to the keV-MeV prompt emission by about 300-400 s. The decay time of this flare is shorter than the duration of the flare (500 s) or its delay.

Conclusions. While we cannot offer a straightforward explanation, we discuss the implications of the flare properties and suggest ways toward understanding it.
\end{abstract}

Key words. gamma rays: stars - radiation mechanisms: non-thermal

\section{Introduction}

Optical/near-infrared (NIR) emission from gamma-ray burst (GRB) sources is very diverse. Rapid optical observations have shown that the canonical power law decay is preceded by a rising part (e.g., Rykoff et al. 2004; Oates et al. 2009; Melandri et al. 2010). On top of this, some afterglows have shown substantial optical variability, both at early times as well as at late times. The early ones can be resolved into a component which tracks the prompt gamma-rays (e.g., GRB 041219A: Vestrand et al. 2005; Blake et al. 2005; GRB 050820A: Vestrand et al. 2006; GRB 080319B: Racusin et al. 2008) and an afterglow component which starts during or shortly after the prompt phase (e.g., GRB 990123: Akerlof et al. 1999, GRB 030418: Rykoff et al. 2004, GRB 060111B: Klotz et al. 2006, GRB 121217A: Elliott et al. 2014). The former component has been attributed to internal shocks (Doi et al. 2007), while the latter component has

\footnotetext{
* Partly based on observations collected at the European Organisation for Astronomical Research in the Southern Hemisphere under ID 092.A-0231(B).

$\star \star$ The GROND photometry table is only available at the CDS via anonymous ftp to cdsarc.u-strasbg. fr (130.79.128.5) or via http://cdsarc.u-strasbg.fr/viz-bin/qcat?]/A+A/568/A75 $\star \star \star$ Fellow of the Alexander v. Humboldt Foundation.
}

been interpreted as reverse shock emission, e.g., Sari \& Piran (1999); Meszaros \& Rees (1999) or residual shell collisions at larger radii (Li \& Waxman 2008). Alternative models include a different, optically thin emission region (Fan et al. 2009), or dust destruction (Cui et al. 2013). At intermediate times, a large fraction of GRBs has been found to exhibit optical flares during the first $1000 \mathrm{~s}$ (Swenson et al. 2013). At late times, some GRB afterglows (021004, 030329, Lipkin et al. 2004) showed bumps on top of the canonical fading, on timescales of $10^{4}$ $10^{6} \mathrm{~s}$. Originally, these bumps were interpreted as the interaction of the fireball with moderate density enhancements in the ambient medium, with a density contrast of order 10 (Lazzati et al. 2002), and later by additional energy injection episodes (Björnsson et al. 2004; de Ugarte Postigo et al. 2005).

GROND, a simultaneous 7-channel optical/NIR imager (Greiner et al. 2008) mounted at the $2.2 \mathrm{~m}$ telescope of the MaxPlanck-Gesellschaft (MPG), operated by MPG at the European Southern Observatory (ESO) La Silla Observatory (Chile), started operation in May 2007. GROND was built as a dedicated GRB and transient follow-up instrument and has observed basically every GRB visible from La Silla (weather allowing) since April 2008. GROND observations of GRBs within the first day are fully automated (see Greiner et al. 2008 for more details). The spectral energy distribution (SED) obtained with GROND 


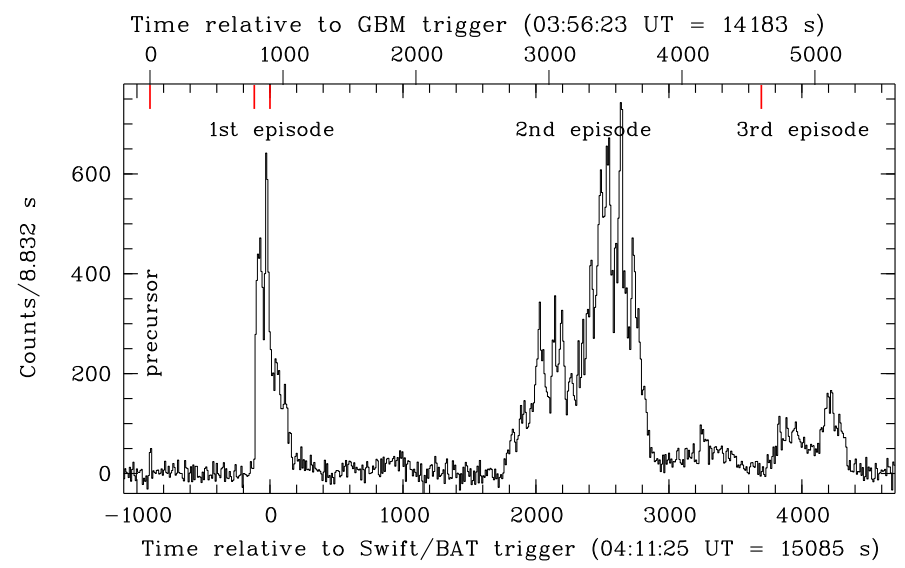

Fig. 1. Background-subtracted light curve of the prompt emission of GRB $130925 \mathrm{~A}$ as measured with Konus-Wind in the $26-1480 \mathrm{keV}$ band. The red, thick vertical lines at the top indicate, in temporal sequence from the left, the first GBM trigger, the second GBM trigger, the BAT trigger and the MAXI trigger.

between 400-2400 nm allows us to not only find high- $z$ candidates (Greiner et al. 2009a; Krühler et al. 2011), but also to measure the extinction and the power law slope (Greiner et al. 2011) with good accuracy.

GRB 130925A triggered the Gamma-ray Burst Monitor (GBM, Meegan et al. 2008) on the Fermi satellite first at 03:56 UT on 25 September 2013 on what seems to be a precursor, and a second time at 04:09 UT (Fitzpatrick 2013). Subsequently, the Burst Alert Telescope (BAT) on the Swift satellite (Gehrels et al. 2004) triggered at $T_{0}$ (BAT) $=$ 04:11:25 UT = 15085 s (trigger = 571 830; Lien et al. 2013). Also, INTEGRAL SPI/ACS (Savchenko et al. 2013) and MAXI/GSC (Suzuki et al. 2013) triggered on this GRB and Konus-Wind detected it in waiting mode (Golenetskii et al. 2013). It was also observed by Mars Odyssey and MESSENGER in the interplanetary network, and these observations strengthen the case for a common origin for all of the emission episodes (Hurley et al. 2013). The overall Konus-Wind light curve of this particularly long burst, unaffected by Earth occultations, is shown in Fig. 1, with the GBM, BAT and MAXI trigger times labelled.

The Swift satellite slewed immediately upon the BAT trigger and started taking data with the XRT and UVOT telescopes at $147 \mathrm{~s}$ after the trigger. A bright X-ray source was found at RA $(2000.0)=02^{\mathrm{h}} 44^{\mathrm{m}} 42^{\mathrm{s}} .4$, Dec $(2000.0)=-26^{\circ} 09^{\prime} 16^{\prime \prime}$ with an error radius of $5^{\prime \prime} 11$ (Lien et al. 2013). UVOT did not detect any obvious emission, but with GROND a very red source was detected (Sudilovsky et al. 2013a). Subsequent spectroscopy with UVES (Vreeswijk et al. 2013) and X-Shooter (Sudilovsky et al. 2013b) revealed multiple emission lines, suggestive of a host galaxy redshift of $z=0.347$. Using standard cosmology $\left(H_{\mathrm{o}}=70 \mathrm{~km} \mathrm{~s}^{-1} \mathrm{Mpc}, \Omega_{\mathrm{M}}=0.27, \Omega_{\Lambda}=0.73\right)$, this implies a luminosity distance of $0.57 \times 10^{28} \mathrm{~cm}$.

The initial Swift/XRT observations show extremely rapid and dramatic flaring, which prompted speculations that this event could be a tidal disruption event (TDE; Burrows et al. 2013), similar to the highly variable soft X-ray light curve observed from Swift J1644+57 (GRB 110328A; Burrows et al. 2011; Levan et al. 2011). Late-time HST observations show that the transient emission is slightly offset from the nucleus of the galaxy by about $0{ }^{\prime} 12(\approx 600 \mathrm{pc}$ in projection), thus arguing against a TDE unless the galaxy contains more than one super-massive black hole (Tanvir et al. 2013). Also, the latetime $\left(>10^{5} \mathrm{~s}\right) \mathrm{X}$-ray behaviour is markedly different from the spiky light curve of Swift J1644+57 and resembles that of a GRB X-ray afterglow, so we adopt the GRB classification in this paper. It remains true, though, that GRB 130925A is among the longest-duration GRBs measured so far, prompting concerns about the maximum possible duration of the central engine in the standard GRB paradigm (Zhang et al. 2014).

Here, we describe observations of the second emission peak, motivated by the GROND data during this time interval. Given that both the Swift and the Fermi satellites were Earth-blocked during most of the GROND observations, we concentrate here on the $\gamma$-ray data of the Konus-Wind and INTEGRAL SPI/ACS instruments, which provide simultaneous coverage. We have checked RHESSI data, but found it to only exhibit background fluctuations for this second emission period (D. Smith, priv. comm.). In the following, our multi-wavelength observations of GRB 130925A are presented in Sect. 2, and an explanation of the data is proposed in Sect. 3. Throughout this paper, we use the definition $F_{v} \propto t^{-\alpha} v^{-\beta}$ where $\alpha$ is the temporal decay index, and $\beta$ is the spectral slope.

\section{Observations}

\subsection{Konus-Wind data}

The Konus-Wind instrument (KW, Aptekar et al. 1995) is a $\gamma$-ray spectrometer consisting of two identical detectors, S1 and S2, which observe the southern and northern ecliptic hemispheres, respectively. GRB 130925A was observed as a count rate increase in the $\mathrm{S} 1$ detector. Thanks to the remote KW orbit around the Lagrangian point L1, with stable background and lack of Earth or planet occultations, the instrument was able to measure the burst's prompt emission for more than 5000 s. A more detailed description of the KW observation of GRB 130925A can be found in Evans et al. (2014).

Since KW did not trigger on any of the various peaks, the data are available only in "waiting mode". In this regime, count rates with a coarse time resolution of $2.944 \mathrm{~s}$ are recorded in three energy bands: G1 (26-99 keV), G2 (99-394 keV), and G3 (394-1480 keV). The light curve of the event in the combined G1+G2+G3 energy bands (Fig. 1) starts with the weak precursor at $\sim T_{0}$ (BAT) $-900 \mathrm{~s}$ and thereafter shows several multi-peaked pulses, separated by long periods of low-level emission.

The time history recorded in the three KW energy bands can be considered as a continuous three-channel spectrum covering the 26-1480 keV energy range. After subtracting the background emission as estimated by a polynomial fit from before the precursor to well after the third emission period, we measure the following fluences (all in the standard $\mathrm{KW}$ 20-10000 keV band) for the different emission episodes based on cut-off powerlaw fits to the spectra: 1st major episode (14961-15264 s UT): $9.5 \pm 0.7 \times 10^{-5} \mathrm{erg} \mathrm{cm}^{-2}$; 2nd major episode $\left(16766-17964 \mathrm{~s}\right.$ UT): $3.9 \pm 0.1 \times 10^{-4} \mathrm{erg} \mathrm{cm}^{-2}$; $3 \mathrm{rd}$ major episode $(18768-19463 \mathrm{~s} \mathrm{UT}): 5.6 \pm 0.9 \times 10^{-5} \mathrm{erg} \mathrm{cm}^{-2}$. The total fluence, which accounts also for the weaker interpulse emission, is $6.2 \pm 0.3 \times 10^{-4} \mathrm{erg} \mathrm{cm}^{-2}$. At $z=0.347$ and standard cosmology $\left(H_{\mathrm{o}}=70 \mathrm{~km} \mathrm{~s}^{-1} \mathrm{Mpc}, \Omega_{\mathrm{M}}=0.27\right.$, $\Omega_{\Lambda}=0.73$ ), this implies an isotropic equivalent energy release of $E_{\text {iso }}=1.9 \pm 0.1 \times 10^{53} \mathrm{erg}$. This is one of the most energetic lowredshift GRBs: to our knowledge it is \#2 after GRB 130427A (Maselli et al. 2014). As it will be important in the later discussion, we also provide the individual contributions of the main 
Table 1. Secondary standards (all in $A B$ magnitudes) used for the GROND data.

\begin{tabular}{cccccccc}
\hline \hline Filter & Star I & Star II & Star III & Star IV & Star V & Star VI & Star VII \\
& $02: 44: 44.69$ & $02: 44: 35.90$ & $02: 44: 36.52$ & $02: 44: 34.38$ & $02: 44: 35.89$ & $02: 44: 49.04$ & $02: 44: 42.80$ \\
& $-26: 07: 57.0$ & $-26: 09: 07.3$ & $-26: 08: 09.3$ & $-26: 07: 27.2$ & $-26: 07: 26.9$ & $-26: 09: 28.8$ & $-26: 07: 15.4$ \\
\hline$g^{\prime}$ & $17.48 \pm 0.04$ & $17.65 \pm 0.04$ & $21.95 \pm 0.07$ & $18.38 \pm 0.04$ & $21.05 \pm 0.06$ & $20.82 \pm 0.05$ & $22.76 \pm 0.08$ \\
$r^{\prime}$ & $17.06 \pm 0.04$ & $17.24 \pm 0.04$ & $20.33 \pm 0.05$ & $16.92 \pm 0.04$ & $19.45 \pm 0.05$ & $19.29 \pm 0.04$ & $21.30 \pm 0.07$ \\
$i^{\prime}$ & $16.90 \pm 0.04$ & $17.11 \pm 0.04$ & $18.71 \pm 0.04$ & $16.02 \pm 0.04$ & $17.87 \pm 0.05$ & $18.20 \pm 0.05$ & $19.84 \pm 0.06$ \\
$z^{\prime}$ & $16.85 \pm 0.04$ & $17.04 \pm 0.04$ & $18.03 \pm 0.04$ & $15.60 \pm 0.04$ & $17.12 \pm 0.04$ & $17.72 \pm 0.05$ & $19.19 \pm 0.06$ \\
\hline Filter & Star 1(=I) & Star 2 & Star 3 & Star 4 (=IV) & Star 5(=V) & Star 6 & Star 7 \\
& $02: 44: 44.69$ & $02: 44: 40.97$ & $02: 44: 51.60$ & $02: 44: 34.38$ & $02: 44: 35.89$ & $02: 44: 42.60$ & $02: 44: 51.73$ \\
& $-26: 07: 57.0$ & $-26: 11: 31.8$ & $-26: 08: 53.9$ & $-26: 07: 27.2$ & $-26: 07: 26.9$ & $-26: 10: 08.4$ & $-26: 08: 24.0$ \\
\hline$J$ & $17.03 \pm 0.05$ & $16.34 \pm 0.05$ & $18.71 \pm 0.07$ & $15.44 \pm 0.05$ & $16.71 \pm 0.05$ & $18.89 \pm 0.07$ & $18.22 \pm 0.06$ \\
$H$ & $17.13 \pm 0.06$ & $16.13 \pm 0.05$ & $18.51 \pm 0.08$ & $15.26 \pm 0.05$ & $16.50 \pm 0.05$ & $18.56 \pm 0.08$ & $18.02 \pm 0.07$ \\
$K_{\mathrm{s}}$ & $17.50 \pm 0.08$ & $16.36 \pm 0.07$ & $18.31 \pm 0.09$ & $15.49 \pm 0.07$ & $16.70 \pm 0.08$ & $18.49 \pm 0.10$ & $17.90 \pm 0.08$ \\
\hline
\end{tabular}

Notes. For the NIR, different standards had to be used due to the brightness of the GRB afterglow.

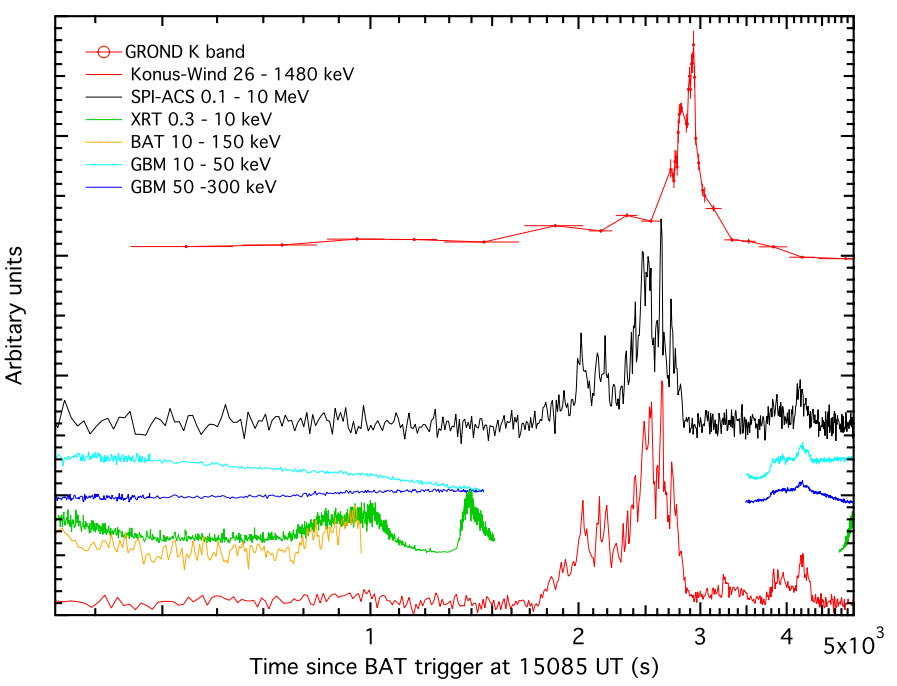

Fig. 2. Light curve of GRB 130925A as observed with various instruments (see labels) at different times. The gap at $\approx 1.6-3.5 \mathrm{ks}$ for GBM and Swift/XRT is due to Earth blockage. The top line shows the $K_{\mathrm{s}}$ light curve as measured with GROND at the same time axis as the gammaray emission.

emission peaks as follows: $2.9 \pm 0.2 \times 10^{52} \mathrm{erg}$ for the first and $1.2 \pm 0.04 \times 10^{53} \mathrm{erg}$ for the second major emission interval.

\subsection{INTEGRAL SPI/ACS data}

The SPI-ACS data were taken from the Integral Data Centre. The original $50 \mathrm{~ms}, 0.1-10 \mathrm{MeV}$ light curve was rebinned into $8 \mathrm{~s}$ temporal bins as shown in Fig. 2, but analyzed at their finest time resolution for the minimum variability timescale.

\subsection{GROND and VLT observations}

GROND exposures automatically started $350 \mathrm{~s}$ after the arrival of the Swift/BAT trigger. Simultaneous imaging in $g^{\prime} r^{\prime} i^{\prime} z^{\prime} J H K_{\mathrm{s}}$ continued for nearly $6 \mathrm{hrs}$, with the last exposures done only in the NIR channels due to morning twilight (after 09:36 UT). Further observations were done in the mornings of Sep. 26, 27 and 29, with one late-time deep host imaging on Oct. 6. GROND data have been reduced in the standard manner (Krühler et al. 2008) using pyraf/IRAF (Tody 1993; Küpcü Yoldaş et al. 2008b). The optical/NIR imaging was calibrated against the

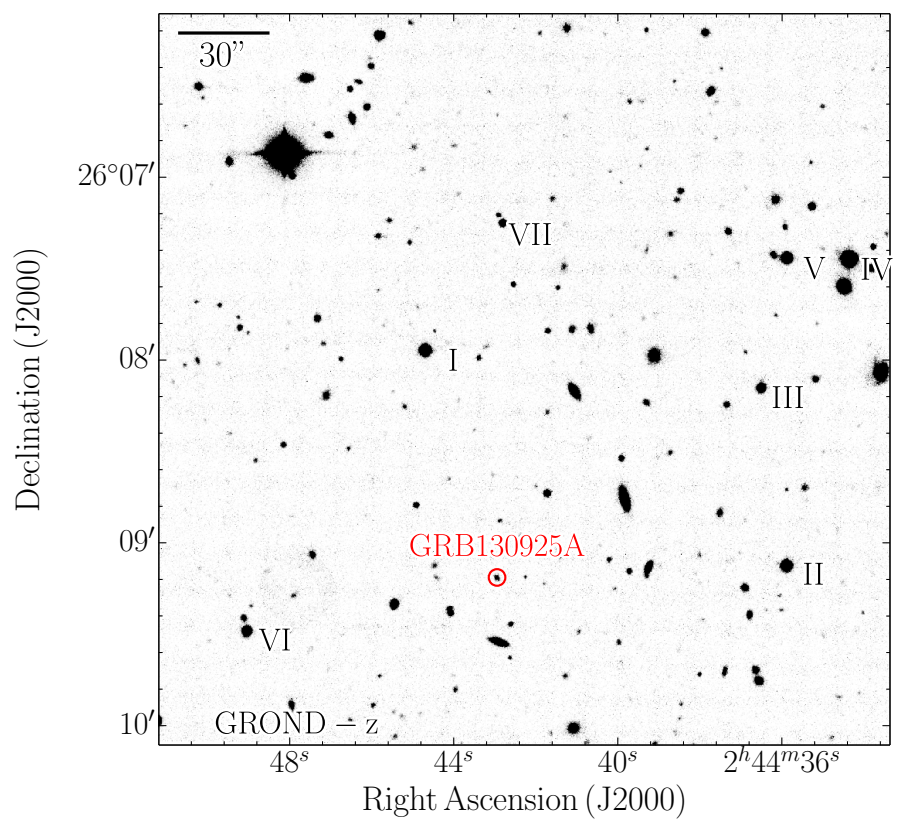

Fig. 3. GROND $z^{\prime}$-band finding chart of GRB 130925A, including the photometric comparison stars (roman letters) for $g^{\prime} r^{\prime} i^{\prime} z^{\prime}$ and partly for $J H K_{\mathrm{s}}$ as detailed in Table 1 (some comparison stars for $J H K_{\mathrm{s}}$ are outside the field as shown here). North is up, and east to the left.

primary SDSS ${ }^{1}$ standard star network, or catalogued magnitudes of field stars from the SDSS in the case of $g^{\prime} r^{\prime} i^{\prime} z^{\prime}$ observations, or the 2MASS catalog for $J H K_{\mathrm{s}}$ imaging. This results in typical absolute accuracies of \pm 0.03 mag in $g^{\prime} r^{\prime} i^{\prime} z^{\prime}$ and \pm 0.05 mag in $J H K_{\mathrm{s}}$. Comparison stars are given in Table 1 and labeled in the finding chart of GRB 130925A (Fig. 3).

We find a variable optical/NIR object at position $\operatorname{RA}(2000.0)=02: 44: 42.96, \quad \operatorname{Dec}(2000.0)=-26: 09: 11.2$ $( \pm 0.3)$ which is fully consistent with the Swift/XRT 1'.4 error circle (Swift/XRT repository). We therefore identify this object as the optical/NIR counterpart of GRB 130925A; its light curve in all 7 GROND filters is shown in Fig. 4.

In order to more accurately estimate the contribution of the constant host flux to the early- and late-time light curve, we observed the field of GRB 130925A with the ESO/VLT UT4

1 http://www.sdss.org 


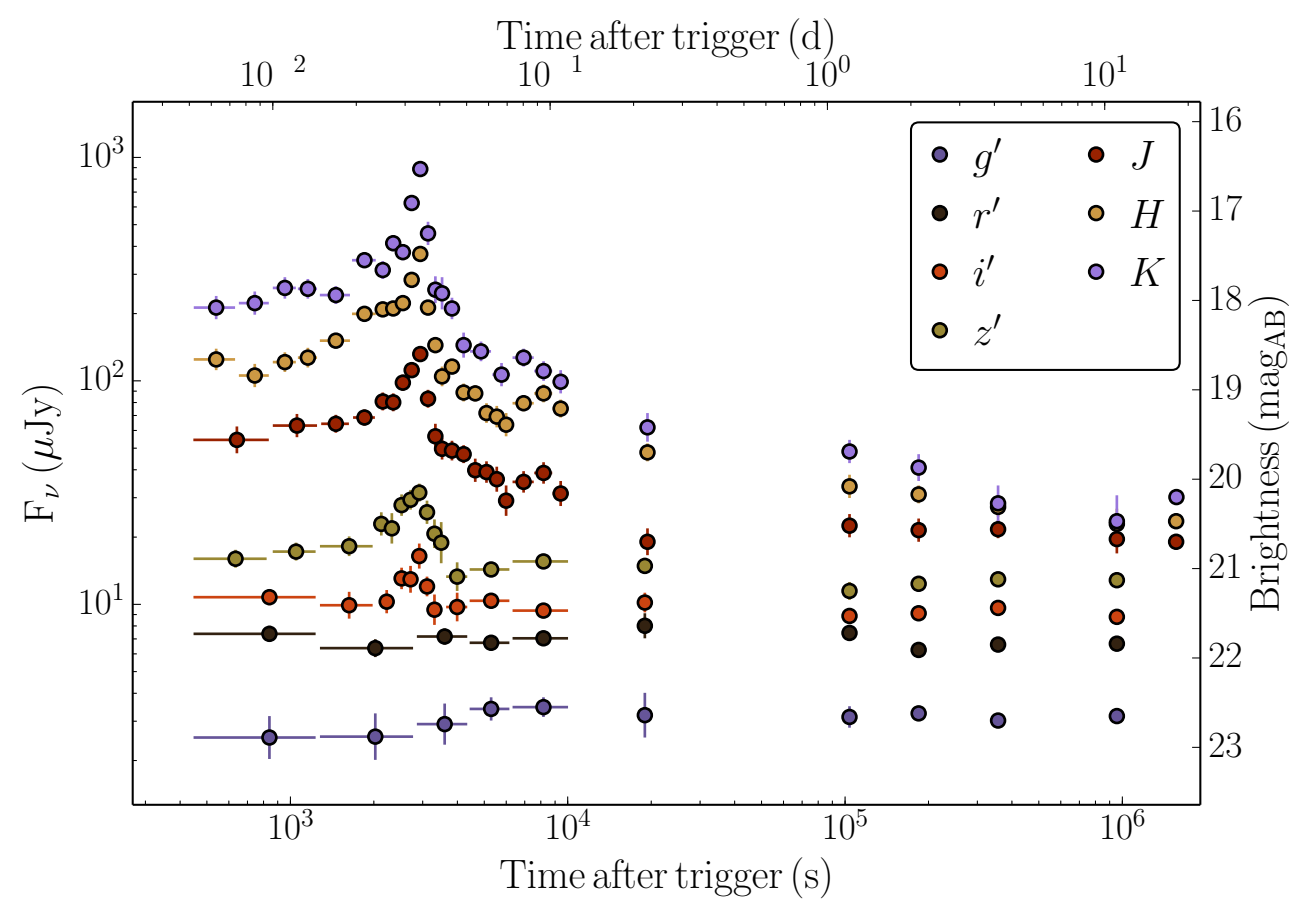

Fig. 4. Light curve of the afterglow of GRB $130925 \mathrm{~A}$ in the 7 GROND filters. The times are relative to the Swift/BAT trigger time. The very last epoch measurements are from HAWK-I observations and represent our best estimate of the host magnitudes. equipped with HAWK-I. Observations started on October 13, 2013, 06:21 UT, which is 18.1 days after the GRB trigger. They were performed under clear sky conditions and consist of sets of dithered exposures with a total integration time of 30,12, 10 and 16 min in the $Y, J, H$ and $K_{\mathrm{s}}$ filter respectively. Data reduction and photometric calibration was performed in a similar fashion as for the GROND data. The measurements are shown as the temporally last data points in Fig. 4.

When comparing to the GROND light curve, the most intriguing feature is the long and clearly visible (Fig. 2) delay of the optical/NIR emission peak relative to the $\gamma$-ray emission (second episode). This delayed optical/NIR emission will be further discussed in Sect. 3.3. below.

\subsection{Swift data}

The Swift/BAT instrument triggered on the first emission episode (not on the precursor which triggered GBM), and follow-up observations with the XRT and UVOT instruments started about $150 \mathrm{~s}$ after the BAT trigger (Lien et al. 2013). While a thorough analysis of all the Swift data has appeared already (Evans et al. 2014), we use some Swift/XRT observations to compare with the GROND data. The corresponding data have been extracted from the Swift/XRT repository (Evans et al. 2009).

\section{Results}

\subsection{Minimum variability time scale of the prompt emission}

The variability in the $\gamma$-ray light curve of GRBs is, in most models, related to a physical origin in the central engine, likely convolved with the bulk Lorentz factor of the outflow. We have estimated the minimum variability time scale (MVT) in GRB $130925 \mathrm{~A}$ with the new method recently developed for high timeresolution GBM data (Bhat 2013). The method is based on the ratio of variances of the GRB emission and the background as a function of the bin width. For the precursor and the first and the third peak as seen by Fermi/GBM, we derive the following MVT: $0.39 \pm 0.02 \mathrm{~s}, 0.92 \pm 0.01 \mathrm{~s}, 6.7 \pm 2.2 \mathrm{~s}$, respectively. For the INTEGRAL/ACS, we obtain $1.10 \pm 0.19 \mathrm{~s}, 4.82 \pm 1.86 \mathrm{~s}$ and $14.2 \pm 5.2 \mathrm{~s}$ for the first, second and third peak, respectively. This shows the typical behaviour of increasing MVT with time. Given that the INTEGRAL/ACS and Fermi/GBM data show good consistency for the first and third peaks, we can be confident in the INTEGRAL-derived MVT value for the second peak, where we have no GBM coverage. Bhat (2013) has estimated the mean MVT for short- and long-duration GRBs as $0.024 \mathrm{~s}$ and $0.25 \mathrm{~s}$, respectively (see his Fig. 3). Thus, the MVT of about $1 \mathrm{~s}$ for the first and about $5 \mathrm{~s}$ for the second peak of GRB 130925A falls in the longest $5 \% / 1 \%$ percentile of the distribution of long-duration GRBs.

If the MVT relates to the bulk Lorentz factor, then GRB 130925A might feature one of the lowest Lorentz factors encountered so far. Applying the formalism of Lithwick \& Sari (2001), and carrying forward the error of the spectral slope to estimate the flux at $1 \mathrm{MeV}$, we obtain minimum Lorentz factors of 37, 20 and 31 for the precursor, first and third peak as seen with Fermi/GBM. We note, though, that using e.g., the $\Gamma_{\mathrm{o}}-E_{\gamma, \text { iso }}$ relation of Liang et al. (2010), we obtain values up to a factor 10 higher.

\subsection{A normal optical/NIR/X-ray afterglow?}

In an attempt to derive a combined Swift/XRT and GROND SED, we have selected time intervals for both instruments which were not affected by flares. Due to the strong flaring activity, Earth blockages of Swift and observability periods of GROND, we are forced to select non-simultaneous, not even overlapping time intervals. Using $36 \mathrm{ks}$ of Swift/XRT data from 100-370 ks after the trigger (to avoid pile-up in the PC-mode data, but still before the light curve break) and GROND data from 3.5-6 ks, we perform a simultaneous spectral fit after re-adjusting the relative normalisation according to the common $t^{-0.9}$ intensity decay. Since the X-ray spectral slope is substantially steeper than the extinction-corrected optical/NIR slope, we employ a broken power law. In contrast to the typical GRB afterglow SEDs (e.g., Greiner et al. 2011) a fit with a fixed slope difference of 


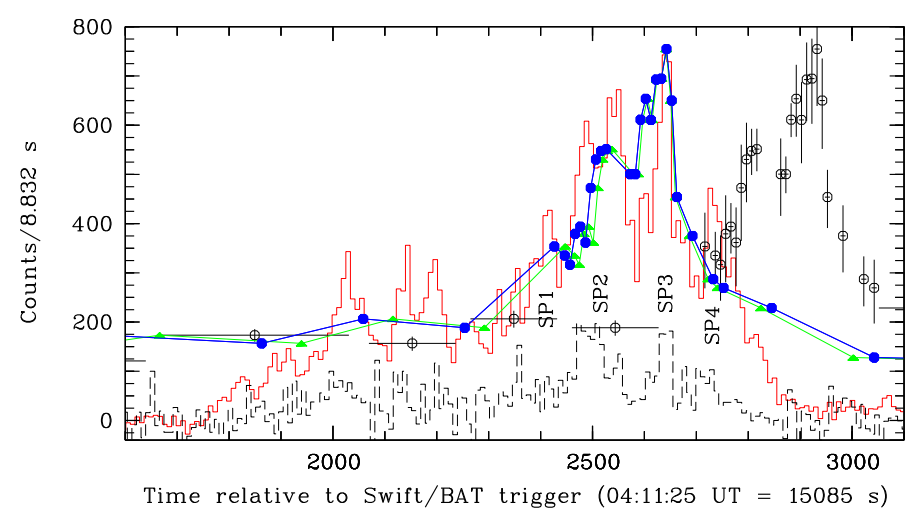

Fig. 5. Expanded view of the delay of the GROND $K_{\mathrm{s}}$-band light curve (open black symbols with error bars) with respect to the KW light curve (red line). In blue and green we show the shifted (-290 s) and stretched (time axis divided by 1.11) GROND $K_{\mathrm{s}}$-band light curve, respectively. The black dashed curve at the bottom is the KW highest energy channel G3 (at a factor 3 stretched intensity scaling).

0.5 between the optical/NIR and X-ray part is rejected at high confidence. Fixing the redshift and the galactic foreground absorption of $N_{\mathrm{H}}^{\mathrm{Gal}}=1.66 \times 10^{20} \mathrm{~cm}^{-2}($ Kalberla 2005) we obtain the following best-fit parameters: $N_{\mathrm{H}}^{\text {Host }}=(1.5 \pm 0.1) \times 10^{22} \mathrm{~cm}^{-2}$, $\beta_{\text {opt } / \mathrm{NIR}}=0.32 \pm 0.03, \beta_{\mathrm{X}}=1.6 \pm 0.1, E_{\text {break }}=1.68 \pm 0.09 \mathrm{keV}$, and $E(B-V)=2.24 \pm 0.26 \mathrm{mag}$ for SMC-like dust, at a reduced $\chi^{2}=0.87$ for 84 degrees of freedom. While formally an acceptable fit, this is incompatible on physical grounds with any previous afterglow modelling, and supports earlier conclusions that suggested a different origin of the X-ray emission, i.e., as dust-scattered prompt flux (Evans et al. 2014) or thermal emission from a hot cocoon (Piro et al. 2014).

\subsection{The delayed optical/NIR peak wrt. gamma-rays}

\subsubsection{Temporal properties}

In the GROND data we see a very sudden increase in brightness at around $2.5 \mathrm{ks}$ after the BAT trigger, followed by a rapid decline after the peak at $3 \mathrm{ks}$, followed by a more gentle decline beyond about $4 \mathrm{ks}$. There is a pronounced dip around $6 \mathrm{ks}$ with another emission peak at $8 \mathrm{ks}$. Fitting a two-component model with a triangular-shaped flare on top of a gentle, smoothly-broken powerlaw (as used above) for the times before and well after the flare we derive a rise slope of $t^{+2.0 \pm 0.3}$ and a decay slope of $t^{-6 \pm 1}$, assuming a $T_{\mathrm{o}}$ at the beginning of the second gamma-ray pulse.

As already mentioned, the most intriguing feature of GRB $130925 \mathrm{~A}$ is the long and clearly visible (Fig. 2) delay of the optical/NIR emission peak relative to the $\gamma$-ray emission (second episode). Such lags have been seen in previous GRBs, though the present delay is surprisingly long: we measure a delay of the optical/NIR peak of $3040 \pm 30$ s relative to the maximum of the first gamma-ray episode, and $405 \pm 30$ s relative to the maximum of the second gamma-ray episode.

When split into single $10 \mathrm{~s}$ integrations around the peak (only possible in the $K_{\mathrm{s}} / H$-bands), we find some substructure in the peak (Fig. 5). To some extent, this substructure reflects the subpulse structure in the KW light curve, in particular the first three sub-pulses (SP1-SP3 in Fig. 5). However, other features are missing or are less pronounced: the fourth peak (SP4) is completely missing, the width of SP3 is much broader in $K_{\mathrm{s}}$ than in gamma-rays, while that of SP2 is narrower. Also, the smaller peaks at 2000-2200 s after the BAT trigger are hardly seen at all in $K_{\mathrm{s}}$. Moreover, a simple shift of the GROND $K_{\mathrm{s}}$-band light curve (blue line in Fig. 5) is a better match than a timestretch (green line). We also note that the best-fit shift in Fig. 5 is by $-290 \mathrm{~s}$, thus matching the peak of the $K_{\mathrm{s}}$-band light curve with the sharp spike SP3, while the above mentioned shift of $405 \pm 30$ s matches the highest fluence peak SP2. We note the interesting property of the optical/NIR flare, namely that its decay time of $\sim 20 \mathrm{~s}$ is much shorter than both the flare duration $(\sim 500 \mathrm{~s})$ and its delay after the second peak ( 300-400 s). The implications of this are discussed further below.

\subsubsection{The spectral energy distribution}

The measured 5 s peak magnitude in the $K$-band is 15.95 (AB) or 14.09 (Vega), and after correction of the host-intrinsic extinction amounts to $K^{\text {corr }}$ (Vega) $=13.5$ mag. This corresponds to a peak luminosity just in the $K$ band of $3 \times 10^{45} \mathrm{erg} \mathrm{s}^{-1}$. The energy release of the main peak ( $250 \mathrm{~s}$ duration) is about $7 \times 10^{48} \mathrm{erg}$ in the $400-2400 \mathrm{~nm}$ band.

The spectral energy distribution of the flare emission as measured with GROND is very steep, but clearly curved, suggesting substantial extinction. A powerlaw fit with SMC-like extinction results in an extinction $A_{\mathrm{V}}=5.0 \pm 0.7 \mathrm{mag}$ and a power law spectral slope of $\beta_{\mathrm{opt}}=1.3 \pm 0.4$. The relatively large errors stem from the correlation between these two parameters, see Fig. 6 . In any case, GRB 130925A is being extinguished by one of the highest dust columns ever measured (e.g., Greiner et al. 2011).

In an attempt to possibly distinguish canonical afterglow emission from that of the optical/NIR peak, we have performed a time-resolved spectral fit of the GROND data using the same time bins as for the combined GROND-KW spectral fits (see below, and Table 2). With the extinction $A_{\mathrm{V}}$ fixed, the resulting best-fit spectral index $\beta$ is shown in the bottom panel of Fig. 6 . We find that the spectral slope during the optical/NIR peak is steeper by at least 0.3 at the $2 \sigma$ level.

\subsubsection{Combined GROND and KW spectral fitting}

Since the GROND data have less flexibility in rebinning than the $\mathrm{KW}$ data, we re-binned the $\mathrm{KW}$ data into time bins as determined by the GROND exposures. We define 11 time slices as given in Table 2. Using the method described in Sect. 2.1, we extracted a set of background-subtracted 3-channel spectra for these 11 time slices, and created a corresponding detector response matrix using standard KW tools.

We then performed a combined fit of the 3-channel KW spectra and the 3-5 (depending on significant excess emission above the host galaxy) filter GROND SED by employing a Band function (Band et al. 1993). Fixing the redshift, and requiring the same extinction in all 11 fits, the resulting best-fit parameters are also shown in Table 2 . While the fits are statistically acceptable (reduced $\chi^{2}=34.1 / 34$ for 79 bins and 34 degrees of freedom for the combined fit of all 11 spectra together), the resulting parameters are physically questionable. With a few exceptions, $E_{\text {peak }}$ is always at the low-energy boundary of the G2 channel. Furthermore, the low-energy slope is always close to 1 , with the variations reflecting the relative rise and fall of the opti$\mathrm{cal} / \mathrm{NIR}$ or $\gamma$-ray flux. Thus, while the model mathematically fits, we do not interpret this as evidence that the optical/NIR emission tracks the $\gamma$-ray emission. 

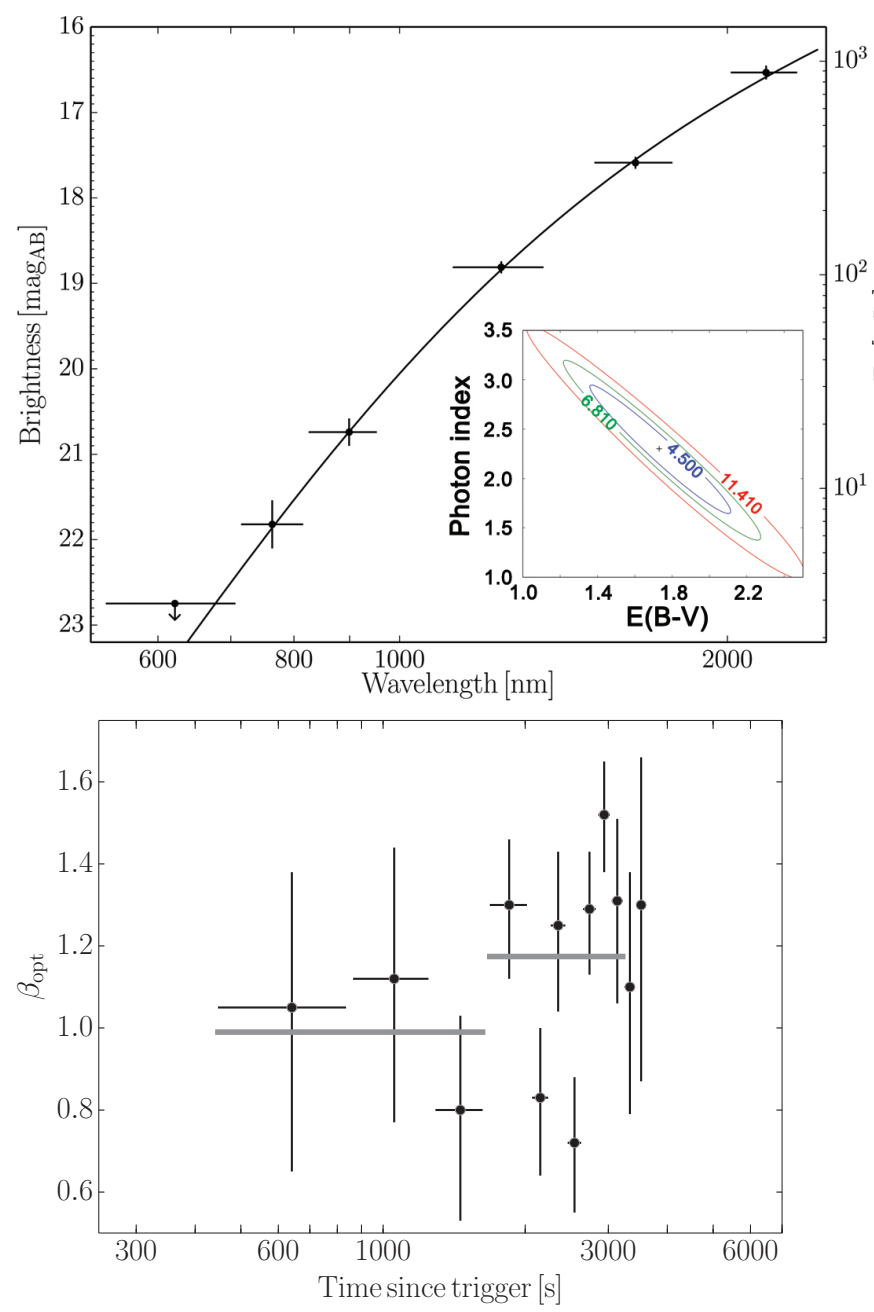

Fig. 6. Top: spectral energy distribution of GRB 130925A during the optical/NIR peak as observed with GROND. The emission of the host galaxy is subtracted. There is no detection in the $g^{\prime} r^{\prime}$-bands above the host emission. The curvature in the SED is due to the strong dust extinction of $A_{V}=5.0 \pm 0.7 \mathrm{mag}$. The inset shows contours of constant $\chi_{\text {red }}$ for the intrinsic spectral slope as a function of reddening (SMC reddening law $\left.A_{V}=2.93 \times E(B-V)\right)$ for the peak. Bottom: evolution of the spectral slope (spectral index $\beta$ which relates to the photon index via $\beta+1$ ) of the optical/NIR afterglow with time with fixed $A_{V}$. For most temporal slices outside the optical/NIR peak, the spectral energy distribution is defined only through $J H K_{\mathrm{s}}$ measurements, and thus the error is relatively large. The horizontal bars visualize the average slope for the peak (right) and the pre-peak (left) period.

\section{Discussion}

Figures 1 and 2 suggest two possible alternative interpretations. In the first, one would argue that the optical/NIR flare has occurred between the second and third gamma-ray emission episodes, and thus is part of the prompt emission, similar to the two X-ray flares before the optical/NIR flare. In this interpretation, one would need to explain why the spectral peak of the various emission episodes changes so drastically between gamma-rays and optical. In a second interpretation, one could consider the optical/NIR flare as delayed emission with respect to the second gamma-ray episode, in which case the challenge is to explain both the delay as well as the fast decay time. We discuss each of these options below.

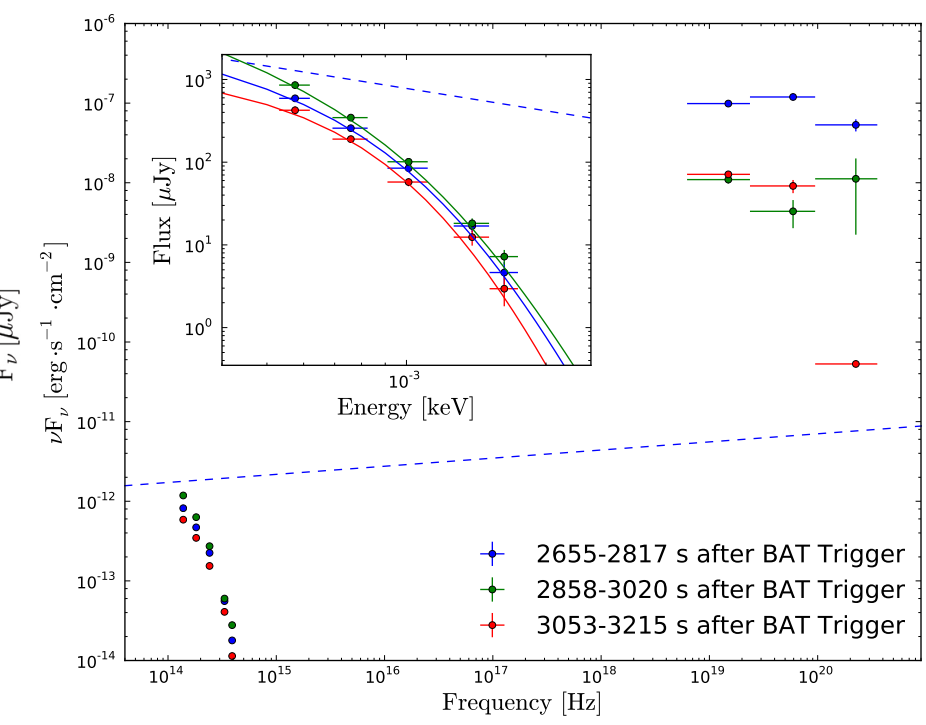

Fig. 7. Broad-band SED at the three epochs 5, 6, and 7 during the optical/NIR flare as seen with GROND (left part) when the prompt flux as seen by $\mathrm{KW}$ (right part) is tailing off into background emission. The inset shows a zoom-in to the GROND data and the best-fit powerlaw model (dashed: un-extinguished power law; solid: power law after extinction).

\subsection{The optical/NIR flare as part of the prompt emission}

\subsubsection{Hard-to-soft evolution}

Analysis of energy-resolved gamma-ray light curves typically shows a generic hard-to-soft evolution of peaks throughout a GRB, manifesting in different features: (i) later peaks have smaller peak energies; (ii) soft energies lag hard energies (Cheng et al. 1995; Norris et al. 2000); (iii) the duration $T_{90}$ gets larger with energy according to $E^{-0.4}$ (Bissaldi et al. 2011). In the case of GRB 130925A, the optical/NIR peak shows a delay which is consistent with a $E^{-0.4}$ dependence, but the widths of the peaks (or $T_{90}$ ) certainly do not. We therefore conclude that the observed optical/NIR peak is likely not a manifestation of the hardto-soft evolution of GRB pulses, despite some similarity in the substructure of the emission.

\subsubsection{A canonical flare}

Looking at Fig. 8 shows that the observed optical/NIR flare is temporally just inbetween major X-ray flares as seen by Swift/XRT. However, the optical/NIR flare seen with GROND is very unlikely to be the optical "counterpart" of a missed X-ray flare, because the times of three X-ray flares seen at $1,2 \mathrm{ks}, 5 \mathrm{ks}$ and $7 \mathrm{ks}$ are covered by GROND data, and show no sign of flux enhancement. Also, the rise and decay times are different, namely $\sim 500 \mathrm{~s}$ in X-rays as compared to $20-30 \mathrm{~s}$ in the optical/NIR.

\subsubsection{Reverse shock}

The reverse shock is predicted to happen with little delay with respect to the gamma-ray emission unless the Lorentz factor is very small. The peak of the emission occurs when the reverse shock crosses the blast wave shell (Meszaros \& Rees 1997; Kobayashi 2000). The corresponding optical emission has a rise time power law index of +0.5 for both a wind and constant density profile (except for the hypothetical case where 
Table 2. Sequence of KW spectra and results of joint GROND-KW spectral fits.

\begin{tabular}{ccccccc}
\hline \hline Spectrum & $\begin{array}{c}\text { Start UT } \\
(\mathrm{s})\end{array}$ & $\begin{array}{c}\text { Stop UT } \\
(\mathrm{s})\end{array}$ & Alpha & Beta & $\begin{array}{c}E_{\text {peak }} \\
(\mathrm{keV})\end{array}$ & $\begin{array}{c}\text { Norm } \\
\left(10^{-3} \mathrm{ph} / \mathrm{cm}^{2} / \mathrm{s} / \mathrm{keV}\right)\end{array}$ \\
\hline 1 & 16754.190 & 17113.358 & $1.08 \pm 0.01$ & $2.38 \pm 0.25$ & $106 \pm 25$ & $5.5 \pm 0.4$ \\
2 & 17154.574 & 17319.438 & $1.00 \pm 0.01$ & $2.64 \pm 0.22$ & $114 \pm 11$ & $14.6 \pm 0.6$ \\
3 & 17351.822 & 17513.742 & $0.99 \pm 0.01$ & $2.45 \pm 0.14$ & $107 \pm 10$ & $18.3 \pm 0.7$ \\
4 & 17546.126 & 17710.990 & $0.94 \pm 0.01$ & $2.44 \pm 0.07$ & $117 \pm 5$ & $35.7 \pm 0.8$ \\
5 & 17740.430 & 17902.350 & $1.01 \pm 0.01$ & $2.66 \pm 0.17$ & $110 \pm 8$ & $20.9 \pm 0.7$ \\
6 & 17943.566 & 18105.486 & $1.22 \pm 0.03$ & $4.03 \pm 52.5$ & $56 \pm 61$ & $2.4 \pm 0.9$ \\
7 & 18137.870 & 18299.790 & $1.15 \pm 0.02$ & $9.38 \pm 14.8$ & $72 \pm 35$ & $2.8 \pm 0.6$ \\
8 & 18335.118 & 18499.982 & $1.03 \pm 0.05$ & $4.72 \pm 33.7$ & $48 \pm 52$ & $7.4 \pm 3.8$ \\
9 & 18523.534 & 18688.398 & $0.98 \pm 0.02$ & $3.72 \pm 3.31$ & $92 \pm 19$ & $9.4 \pm 0.9$ \\
10 & 18729.614 & 19088.782 & $1.06 \pm 0.02$ & $3.79 \pm 3.66$ & $92 \pm 21$ & $3.9 \pm 0.4$ \\
11 & 19118.222 & 19477.390 & $1.00 \pm 0.02$ & $2.89 \pm 0.68$ & $91 \pm 18$ & $6.1 \pm 0.5$ \\
\hline
\end{tabular}

Notes. Alpha and beta are the photon indices of the Band function, and the normalization is at $100 \mathrm{keV}$. Start and stop times are seconds of the day.

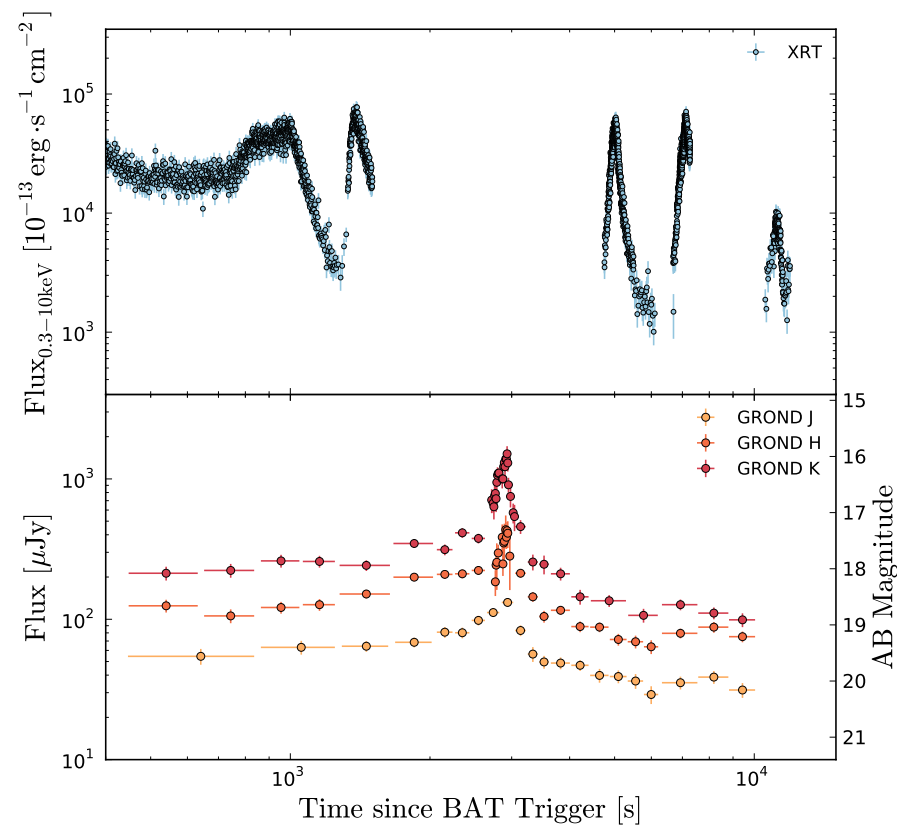

Fig. 8. Swift/XRT X-ray and GROND $J H K_{\mathrm{s}}$ host-subtracted light curve; while the GROND peak is not covered by Swift, the X-ray flares do not show up in the GROND data.

self-absorption is relevant in the optical regime, when the rise can be as fast as +2.5), and a decay time power law index of about -2 for a constant density environment, or up to -3.0 for a wind density profile (Kobayashi 2000; Kobayashi \& Zhang 2003). The decay slopes are clearly inconsistent with our observations. The rise slope could be consistent with the self-absorbed case, but we observe a very sharp peak, so there is no flattening towards a non-self-absorbed phase with a +0.5 rise. We therefore exclude a reverse shock interpretation of the optical/NIR flare in GRB 130925A.

\subsection{The optical/NIR flare as delayed emission}

\subsubsection{Dust destruction}

A number of suggestions have been made in the past which relate the delayed onset of optical/UV emission to the effect of dust in the nearby surrounding medium.

One scenario invokes a dense region around the GRB progenitor, similar to that of a molecular cloud. While the prompt gamma-rays and the X-ray afterglow would pass relatively unaffected, the optical emission would only pass once the dust along our line of sight is completely destroyed through thermal sublimation (e.g. Cui et al. 2013). In the case of GRB 130925A, this scenario is ruled out, because we observe no reduction of the best-fit extinction throughout the burst, to a limit of $\Delta A_{V}<$ $0.4 \mathrm{mag}$.

\subsubsection{Pair-loaded fireball}

Another more promising possibility seems to be the framework of the $\mathrm{e}^{ \pm}$enrichment originally proposed to explain the delayed $\mathrm{GeV}$ flash in Fermi/LAT data (Beloborodov et al. 2014). The prompt $\mathrm{MeV}$ radiation streaming ahead of the blast wave creates a large enrichment in $\mathrm{e}^{ \pm}$which then in turn leads to substantial inverse Compton scattering of the prompt $\mathrm{MeV}$ photons, thus creating a $\mathrm{GeV}$ flash. This $\mathrm{GeV}$ emission starts while the prompt emission is still going on, but lasts longer than the prompt emission because of a broader angular distribution of scattered photons. The model reproduces the delayed onset, the steep rise, the peak flux, the time of the peak, the long smooth decay and the spectral slope of the GeV flash emission (Beloborodov et al. 2014). Simultaneously, a bright optical flash is predicted (Beloborodov et al. 2014), which has been nicely confirmed in the RAPTOR data (Vestrand et al. 2014) of the GRB 130427A afterglow (Vurm et al. 2014).

The delay of the GeV/optical flash is determined by two parameters, the pair-loading radius and the Lorentz factor (Beloborodov et al. 2014). The pair-loading radius is $R_{\text {load }} \approx$ $10^{17} E_{54}^{1 / 2} \mathrm{~cm}$, where $E_{54}$ is the isotropic equivalent energy of the prompt GRB energy ahead of the forward shock. For GRB 130925A, we take $E_{54}$ as the sum of the first prompt pulse and half of the second, i.e. $E_{54}=0.08$, and thus $R_{\text {load }}=$ $2.9 \times 10^{16} \mathrm{~cm}$. Together with the delay of $3040 / 405 \mathrm{~s}$ (relative to first or second episode) we obtain an inferred Lorentz factor of $\Gamma_{405 s} \approx\left((1+z) * R_{\mathrm{load}} / 2 c \delta t\right)^{1 / 2} \sim 40$ or $\Gamma_{3040 s}=11$. It is not obvious which $\gamma$-ray peak to take as reference: One could argue that the delay of the observed optical/NIR peak should be taken relative to the first emission episode, as the blast wave should start immediately after the beginning of the explosion (possibly even at the precursor). However, each prompt emission peak is likely associated with a large energy injection into the blast wave, which may effectively offset the reference time. In any case, the inferred Lorentz factors are of the same order of magnitude as the Lorentz factor derived above from the rising part of the afterglow, and the low absolute value of the inferred Lorentz 
factor is also nicely compatible with the exceptionally long minimum variability time scale.

As a consequence of the low Lorentz factor the corresponding photosphere of the GRB ejecta (Hascoët et al. 2014) implies a photospheric time scale of $1.9 \mathrm{~ms}(\Gamma=40)$ and $1.2 \mathrm{~s}$ $(\Gamma=11)$ for the above two options. The latter Lorentz factor is perfectly consistent with the MVT of one and five seconds as deduced from the prompt emission of the first and second emission episodes.

The only potential mismatch with this pair-loaded fireball scenario is the sub-structure in the flare, as well as its fast decay time scale. It remains to be seen through detailed simulations whether this scenario will work for GRB 130925A.

\subsubsection{Internal dissipation}

Finally, the fast decay of the optical/NIR flare could be considered as evidence for an internal origin within the jet, similar to models for the X-ray flares (but see above Sect. 4.1.). In the standard picture of the blast-wave emission the curvature effect implies that the decay time scale of a flare is of the same order of magnitude as its duration. This can be avoided either by a jet opening angle smaller than $1 / \Gamma$, or by non-isotropic emission in the blast-wave frame (e.g., limb-brightened, Beloborodov et al. 2011). The former case is unlikely, as this GRB shows neither a particularly spiky light curve, nor evidence of particularly high Lorentz factor. In contrast, the latter case in fact also produces a delay of the emission at very small spreading in time, exactly as we observe in GRB 130925A. If this is the true interpretation, GRB 130925A might be the first observational evidence for limb-brightened jet emission.

If the optical/NIR flare and the second gamma-ray peak are indeed related, e.g. by being different episodes of emission from the same shell but at different radii, one would expect the GROND light curve to be stretched rather than only shifted with respect to the second gamma-ray peak (as Fig. 5 demonstrates, both options are consistent with the data, with a preference for a shift). The similarities between the two peaks are then likely the result of the imprint of the outflow geometry or jet structure on the emission, which might be dominated by different processes at the different frequencies.

\subsection{Comparison to previous GRBs}

While there have been very early optical observations covering the prompt gamma-ray emission for about a dozen objects, the combination of sensitivity and time-resolution has in most cases not allowed the establishment of a clear timing pattern of the optical relative to the gamma-ray emission. Apart from GRB 130427A, which is explained in a different way (Vurm et al. 2014), the most obvious cases showing substantial delay of the optical flare relative to the gamma-ray emission are GRB 041219A as observed with PAIRITEL (Blake et al. 2005), one of the other few ultra-long bursts GRB 111209A as observed by TAROT (Klotz et al. 2011; Stratta et al. 2013), and GRB 121217A as observed with GROND (Elliott et al. 2014). In all these cases, the optical flare occurs delayed, but still within the T90 of the prompt gamma-ray emission. Less clear cases wrt delay, though also with clear optical variability, are GRBs 080129 (Greiner et al. 2009b) and 080928 (Rossi et al. 2011). The interpretation of these lags has been diverse: while Blake et al. (2005) argue that the $J H K_{\mathrm{s}}$ emission is consistent with internal shocks, Stratta et al. (2013) suggest two different emission regions, and
Rossi et al. (2011) discuss large-angle emission where the synchrotron peak flux and the peak energy of the electron ensemble are functions of the viewing angle of the observer with respect to the jet axis. GRB 041219 is the only earlier flare which also showed sub-structure, and thus has a similar interpretative problem as our GRB 130925A.

The very long minimum variability time, the relatively faint afterglow with respect to the prompt emission, and the very shallow decay of the afterglow up to very late times suggest a low Lorentz factor, of order 10-20. This is interesting with respect to the range of Lorentz factors of 30-400 recently deduced for a sample of GRBs (Hascoët et al. 2014). This suggests that the range of Lorentz factors could be even broader, with little effect on the prompt GRB spectrum and/or luminosity. We note in passing that this would also be consistent with the photospheric origin of the prompt emission in GRBs (Thompson 1994; Ryde \& Pe'er 2009; Pe'er 2011; Ryde et al. 2011; Giannios 2012; Vurm et al. 2013; Ruffini et al. 2013; Beloborodov 2013).

\section{Conclusions}

We have observed a pronounced optical/NIR flare related to GRB 130925A. Our well-sampled multi-color GROND light curve shows a peak which is delayed by $290 \mathrm{~s}$ with respect to the second $\gamma$-ray peak. This is not dissimilar to several other GRBs for which prompt optical emission has been detected. However, the exquisite data quality for GRB 130925A allows us to exclude the leading contenders. First, given the delay by nearly the full pulse-width, the optical/NIR emission cannot be just the extrapolation of a Band (or any other broad-band model) extension from the $\gamma$-ray emission. Second, the typical interpretation of being due to a reverse shock also does not apply, since the optical/NIR peak in GRB 130925A exhibits a very sharp rise and an extremely steep decay, both much faster than expected for a reverse shock. Other options have been discussed as well, suggesting that the main observational feature of the decay time being shorter than the delay time can, in principle, be explained. However, much more detailed considerations are needed to derive a coherent picture, which are beyond the scope of this paper.

Acknowledgements. We thank the referee, D. Burrows, for a careful reading of the manuscript and his detailed suggestions. J.G. and H.F.Y. acknowledge support by the DFG cluster of excellence "Origin and Structure of the Universe" (www.universe-cluster.de). P.S., J.F.G. and M.T. acknowledge support through the Sofja Kovalevskaja award to P. Schady from the Alexander von Humboldt Foundation, Germany. S.K. and A.N.G. acknowledge support by DFG grant K1 766/16-1. S.S. acknowledges support by the Thüringer Ministerium für Bildung, Wissenschaft und Kultur, FKZ 12010-514. D.A.K. acknowledges TLS Tautenburg for financial support. AvK and XLZ acknowledge support by Space Agency of the Deutsches Zentrum für Luft- und Raumfahrt e.V. (DLR) through funding by the Bundesministerium für Wirtschaft und Technologie under FKZ 50 OG 1101. K.H. is grateful for support under NASA grants NNX13AI54G, NNX11AP96G, and NNX07AR71G. Part of the funding for GROND (both hardware as well as personnel) was generously granted from the Leibniz-Prize to Prof. G. Hasinger (DFG grant HA 1850/28-1). The Konus-Wind experiment is partially supported by a Russian Space Agency contract, RFBR grants 12-0200032a and 13-02-12017 ofi-m. This work made use of data supplied by the UK Swift Science Data Centre at the University of Leicester. Facilities: Max Planck: 2.2 m (GROND), Konus-Wind, INTEGRAL (SPI-ACS), Swift, Suzaku.

\section{References}

Akerlof, C., Balsano, R., Barthelmy, S., et al. 1999, Nature 398, 400 Aptekar, R. L., Frederiks, D. D., Golenetskii, S. V., et al. 1995, SSRv, 71, 265 Band, D., Matteson, J., Ford, L., et al. 1993, ApJ, 413, 281

Blake, C. H., Bloom, J. S., Starr, D. L., et al. 2005, Nature 435, 181 Beloborodov, A. M. 2013, ApJ, 764, 157

Beloborodov, A. M., Daigne, F., Mochkovitch, R., \& Uhm, Z. L. 2011, MNRAS, 410, 2422 
Beloborodov, A. M., Hasoët, R., \& Vurm, I. 2014, ApJ, 788, A36 Bhat, P. N. 2013, in Proc. 7th Huntsville GRB Symp., Huntsville, Apr. 2013, eConf Proc. C1304143

Bissaldi, E., von Kienlin, A., Kouveliotou, C., et al. 2011, ApJ, 733, 97

Björnsson, G., Gudmundsson, E. H., \& Johannesson, G. 2004, ApJ, 615, L77

Blake, C. H., Bloom, J. S., Starr, D. L., et al. 2005, Nature, 435, 181

Burrows, D. N., Kennea, J. A., Ghisellini, G., et al. 2011, Nature, 476, 421

Burrows, D. N., Malesani, D., Lien, A. Y., Cenko, S. B., \& Gehrels, N. 2013, GRB Coordinates Network, Circular Service, 15253

Cheng, L. X., Ma, Y. Q., Cheng, K. S., \& Zhou, Y. Y. 1995, A\&A, 300, 746

Cui, X.-H., Li, Z., \& Xin, L.-P. 2013, RA\&A (IOP Publishing) 13, 57

de Ugarte Postigo, A., Castro-Tirado, A. J., Gorosabel, J., et al. 2005, A\&A, 443, 841

Doi, H., Takami, K., \& Yamazaki, R. 2007, ApJ, 659, L95

Elliott, J., Yu, H.-F., Schmidl, S., et al. 2014, A\&A, 562, A100

Evans, P. A., Beardmore, A. P., Page, K. L., et al. 2009, MNRAS, 397, 1177

Evans, P. A., Willingale, R., Osborne, J. P., et al. 2014, MNRAS, accepted [arXiv: 1403.4079]

Fan, Y., Zhang, B., \& Wei, D. 2009, Phys. Rev., 79, 021301

Fitzpatrick, G. 2013, GRB Coordinates Network, Circular Service, 15255

Gehrels, N., Chincarini, G., Giommi, P., et al. 2004, ApJ, 621, 558

Ghirlanda, G., Nava, L., Ghisellini, G., et al. 2012, MNRAS, 420, 483

Giannios, D. 2012, MNRAS, 422, 3092

Golenetskii, S., Aptekar, R., Frederiks, D., et al. 2013, GRB Coordinates Network, Circular Service, 15260

Greiner, J., Bornemann, W., Clemens, C., et al. 2008, PASP, 120, 405

Greiner, J., Krühler, T., Fynbo, J. P. U., et al. 2009a, ApJ, 693, 1610

Greiner, J., Krühler, T., McBreen, S., et al. 2009b, ApJ, 693, 1912

Greiner, J., Krühler, T., Klose, S., et al. 2011, A\&A, 526, A30

Hascoët, R., Beloborodov, A. M., Daigne, F., \& Mochkovitch, R. 2014, ApJ, 782,5

Hurley, K., Golenetskii, S., Aptekar, R., et al. 2013, GRB Coordinates Network, Circular Service, 15278

Kalberla, P. M. W., Burton, W. B., Hartmann, D., et al. 2005, A\&A, 440, 775

Klotz, A., Gendre, B., Stratta, G., et al. 2006, A\&A, 451, L39

Klotz, A., Gendre, B., Boer, M., \& Atteia, J.-L. 2011, GRB Coordinates Network, Circular Service, 12637

Kobayashi, S. 2000, ApJ, 545, 807

Kobayashi, S., \& Zhang, B. 2003, ApJ, 597, 455

Krühler, T., Küpcü Yoldaş, A., Greiner, J., et al. 2008, ApJ, 685, 376

Krühler, T., Schady, P., Greiner, J., et al. 2011, A\&A, 526, A153

Küpcü Yoldaş, A., Krühler, T., Greiner, J., et al. 2008b, AIP Conf. Proc., 1000, 227

Lazzati, D., Rossi, E., Covino, S., Ghisellini, G., \& Malesani, D. 2002, A\&A, 396, L5

Levan, A. J., Tanvir, N. R., Cenko, S. B., et al. 2011, Science, 333, 199

Li, Z., \& Waxman, E. 2008, ApJ, 674, L65

Liang, E.-W., Yi, S.-Y., Zhang, J., et al. 2010, ApJ, 725, 2209
Lien, A. Y., Markwardt, C. B., Page, K. L., et al. 2013, GRB Coordinates Network, Circular Service, 15246

Lipkin, Y. M., Ofek, E. O., Gal-Yam, A., et al. 2004, ApJ, 606, 381

Lithwick, Y., \& Sari, R. 2001, ApJ, 555, 540

Malesani, D., Cenko, S. B., Burrows, D. N., Evans, P. A., \& Gehrels, N. 2013 GRB Coordinates Network, Circular Service, 15280

Maselli, A., Melandri, A., Nava, L., et al. 2014, Science, 343, 48

Meegan, C., Lichti, G., Bhat, P. N., et al. 2008, ApJ, 702, 791

Melandri, A., Kobayashi, S., Mundell, C. G., et al. 2010, ApJ, 723, 1331

Meszaros, P., \& Rees, M. J. 1997, ApJ, 476, 232

Meszaros, P., \& Rees, M. 1999, MNRAS, 306, L39

Norris, J. P., Marani, G. F., \& Bonnell, J. T. 2000, ApJ, 534, 248

Oates, S. R., Page, M. J., Schady, P., et al. 2009, MNRAS, 395, 490

Pe'er, A. 2011, ApJ, 732, A49

Piro, L., Troja, E., Gendre, B., et al. 2014, ApJ, 790, L15

Racusin, J. L., Karpov, S. V., Sokolowski, M., et al. 2008. Nature, 455, 183

Rossi, A., Schulze, S., Klose, S., et al. 2011, A\&A, 529, A142

Ruffini, R., Siutsou, I. A., \& Vereshchagin, G. V. 2013, ApJ, 772, A11

Ryde, F., \& Pe'er, A. 2009, ApJ, 702, 1211

Ryde, F., Pe'er, A., Nymark, T., et al. 2011, MNRAS, 415, 3693

Rykoff, E., Smith, D. A., Price, P. A., et al. 2004, ApJ, 601, 1013

Sari, R., \& Piran, T. 1999, ApJ, 517, L109

Savchenko, V., Beckmann, V., Ferrigno, C., et al. 2013, GRB Coordinates Network, Circular Service, 15259

Schlegel, D., Finkbeiner, D., \& Davis, M. 1998, ApJ, 500, 525

Stratta, G., Gendre, B., Atteia, J. L., et al. 2013, ApJ, 779, 66

Sudilovsky, V., Kann, D. A., \& Greiner, J. 2013a, GRB Coordinates Network, Circular Service, 15247

Sudilovsky, V., Kann, D. A., Schady, P., et al. 2013b, GRB Coordinates Network, Circular Service, 15250

Suzuki, K., Sakakibara, H., Negoro, H., et al. 2013, GRB Coordinates Network, Circular Service, 15248

Swenson, C. A., Roming, P. W. A., De Pasquale, M., \& Oates, S. R. 2013, ApJ, 774, A2

Tanvir, N. R., Levan, A. J., Hounsell, R., et al. 2013, GRB Coordinates Network, Circular Service, 15489

Thompson, C. 1994, MNRAS, 270, 480

Tody, D. 1993, in Astronomical Data Analysis Software and Systems II, eds. R. J. Hanisch, R. J. V. Brissenden, \& J. Barnes, ASP Conf., 52, 173

Vestrand, W. T., Wozniak, P. R., Wren, J. A., et al. 2005, Nature, 435, 178

Vestrand, W. T., Wren, J. A., Wozniak, P. R., et al. 2006, Nature, 442, 172

Vestrand, W. T., Wren, J., Panaitescu, A., et al. 2014, Science, 343, 38

Vreeswijk, P. M., Malesani, D., Fynbo, J. P. U., De Cia, A., \& Ledoux, C. 2013, GRB Coordinates Network, Circular Service, 15249

Vurm, I., Lyubarsky, Y., \& Piran, T. 2013, ApJ, 764, 143

Vurm, I., Hascoët, R., \& Beloborodov, A. M. 2014, ApJ, 789, L37

Zhang, B.-B., Zhang, B., Murase, K., Connaughton, V., \& Briggs, M. 2014, ApJ, 787,66

Zhao, Y.-N., \& Shao, L. 2014, ApJ, 789, 74 